\title{
On a Regularity Problem Occurring in Connection with Anosov Diffeomorphisms
}

\author{
Jean-Lin Journé` \\ Department of Mathematics, Fine Hall, Washington Road, Princeton University, \\ Princeton, NJ 08544, USA
}

\begin{abstract}
Let $\mathscr{M}$ be a $C^{\infty}$-manifold and $\mathscr{F}_{s}$ and $\mathscr{F}_{u}$ be two Hölder foliations, transverse, and with uniformly $C^{\infty}$ leaves. If a function $f$ is uniformly $C^{\infty}$ along the leaves of the two foliations, then it is $C^{\infty}$ on $\mathscr{M}$. The proof is elementary.
\end{abstract}

It is well known that a function whose restrictions to lines parallel to the coordinate axes are uniformly $\mathscr{C}^{\infty}$ is itself a $\mathscr{C}^{\infty}$-function. The problem amounts to reconstructing mixed derivatives and can be very easily done using Fourier transform.

In studying Anosov diffeomorphisms a similar sort of problem arises. In [LMM] it was observed that, very frequently, one could prove existence of derivatives along the stable and unstable manifolds which are smooth. However, the corresponding foliations are only Hölder, and it is not clear how to reduce to the original case as when the foliations are $\mathscr{C}^{\infty}$. In [LMM] however, the authors succeeded in giving a proof of the global smoothness of their functions by using elliptic theory, as well as another regularity property of their foliations, which is not easy to check [LMM]. We shall present an alternate approach to these sort of results. It won't require the extra regularity condition on the foliations, and therefore will lend itself to other kinds of applications.

We come to the statement of our theorem, which is conjectured in [LMM], at least implicitly.

Let $\mathscr{M}$ be a $\mathscr{C}^{\infty}$-manifold. We shall denote by $\mathscr{F}$ a foliation of $\mathscr{M}$ and for each $M \in \mathscr{M}, \mathscr{L}^{M}$ will be the leaf of $\mathscr{F}$ containing $M$. We assume that the leaves are uniformly $\mathscr{C}^{\infty}$ and that $T^{M}$, the tangent space at $M$ of $\mathscr{L}^{M}$, is a Hölder function of $M$. We now suppose that we have two such foliations which are transverse.

Theorem. If $f: \mathscr{M} \rightarrow \mathbb{R}$ is $\mathscr{C}^{n, \alpha}$ along the leaves of $\mathscr{F}_{\mathrm{s}}$ and $\mathscr{F}_{\text {u }}$ for some $\alpha>0$ uniformly, then $f$ is $\mathscr{C}^{n, \beta}$ for some $\beta>0$.

Corollary. If $f: \mathscr{M} \rightarrow \mathbb{R}$ is $\mathscr{C}^{\infty}$ along the leaves of $\mathscr{F}_{s}$ and $\mathscr{F}_{u}$ uniformly, then $f$ is $\mathscr{C}^{\infty}$.

* Address after June 1986: 5 Rue des Petits-Champs, F-75001 Paris, France 
The theorem will be proved by induction on $n$. The statement for $n$ fixed will be refered to as $\mathscr{P}(n)$.

We should mention that another approach to this problem, involving Fourier transform, and somewhat simpler than the one in [LMM] has been recently found by Katok and Hurder. It also uses some extra regularity on the foliations.

It would be more satisfactory to prove this theorem without any regularity whatsoever on the foliations because the most important assumption seems to be that the foliations are transverse. This is still out of reach.

It is a real pleasure to thank Rafaël de la Llave for bringing these problems to my knowledge and for his encouragements and help.

In Sect. I we present some preliminaries. In Sect. II we prove $\mathscr{P}(1)$ and the induction procedure is detailed in Sect. III.

\section{Preliminaries and Notations}

For each point $M \in \mathscr{M}, \mathscr{L}_{u}^{M}$ and $\mathscr{L}_{s}^{M}$ are the leaves of $\mathscr{F}_{u}$ and $\mathscr{F}_{s}$ respectively, which contain $M$. For two points $M$ and $R$ in $\mathscr{M}$ we shall denote by $P$ and $Q$ the points $\mathscr{L}_{s}^{M} \cap \mathscr{L}_{u}^{R}$ and $\mathscr{L}_{u}^{M} \cap \mathscr{L}_{s}^{R}$.

Notice that because our problem is local we can assume that $\mathscr{M}$ is actually an open set in $\mathbb{R}^{d_{s}+d_{u}}$. For each point $\mathbb{R}^{d_{s}+d_{u}}$ decomposes as $T_{s}^{M}+T_{u}^{M}$. If we let $\Pi_{s}^{M}+\Pi_{u}^{M}$ be the corresponding decomposition of the Identity, we can define $\mathscr{C}^{\infty}$-diffeomorphisms between the leaves of $\mathscr{F}_{u}$ and $M+T_{u}^{M}$ by setting $\tilde{\Pi}_{u}^{M}(R)=M+\Pi_{u}^{M}(\mathbf{M R})$, and similarly between the leaves of $\mathscr{F}_{s}$ and $M+T_{s}^{M}$. If $f$ is a function defined on $\mathscr{M}$, then $f_{P, u}^{M}$ denotes the projection on $M+T_{u}^{M}$ of its restriction to $\mathscr{L}_{u}^{P}$; that is if $R \in \mathscr{L}_{u}^{P}, f(R)=f_{P, u}^{M}\left(\tilde{\Pi}_{u}^{M}(R)\right)$. Similarly for $f_{Q, s^{*}}^{M}$.

The gradient of a function defined on $M+T_{u}^{M}$ is denoted $\nabla_{u}^{M}$; e.g. $\nabla_{u}^{M} f_{P, u}^{M}\left(\widetilde{\Pi}_{u}^{M}(R)\right)$. The gradient at $P$ of a function defined on $\mathscr{L}_{u}^{P}$ is simply denoted $\nabla_{u} ;$ e.g. $\nabla_{u} f(P)$. Similarly for $\nabla_{s}^{M}$ and $\nabla_{s}$.

The geometric properties of the foliations which we shall need are very simple and direct consequences of the fact that they are Hölder and transverse. They are summarized in the following lemma.

Lemma 1. Let $M, P, Q, R$ be as before and suppose $\|\mathbf{M R}\|$ small enough. Then

i) $\|\mathbf{M R}\| \simeq\|\mathbf{M P}\|+\|\mathbf{M Q}\|$.

ii) There exists $\varepsilon>0$ and $\eta>0$ such that if $c\|\mathbf{M P}\|^{1+\eta} \leqq\|\mathbf{M Q}\| \leqq C\|\mathbf{M P}\|^{1 / 1+\eta}$, then $\|\mathbf{M Q}-\mathbf{P R}\|=\|\mathbf{M P}-\mathbf{Q R}\|$ is $O\left(\|\mathbf{M Q}\|^{1+\varepsilon}\right)$ and $O\left(\|\mathbf{M P}\|^{1+\bar{\varepsilon}}\right)$.

We omit the proof of this lemma, which is trivial. Let us mention that while $\alpha, \beta$, $\gamma, \omega, \zeta, v, \mu, \xi$ will denote small non-negative numbers changing from line to line, $\varepsilon$ and $\eta$ will remain fixed.

A crucial ingredient in the proof of the theorem will be the characterization of $\mathscr{C}^{n, \alpha}$ functions in $\mathbb{R}^{d}$ by Companato [C]. We shall state this characterization in the setting of a $\mathscr{C}^{\infty}$-submanifold of $\mathbb{R}^{d}$.

Lemma 2. A function $f$ defined on a $\mathscr{C}^{\infty}$-submanifold $\mathscr{L}$ of $\mathbb{R}^{d}$ is $\mathscr{C}^{n, \alpha}$ on $\mathscr{L}$ for some $n \in \mathbb{N}$ and $\alpha \in] 0,1\left[\right.$, if for each point $M \in \mathscr{L}$, there exists a $\mathscr{C}^{n, \alpha}$ function $f_{M}$ such that $\left\|f_{M}\right\| \widehat{\mathscr{C}^{n, \alpha}} \leqq C$ and

$$
f(N)-f_{M}(N)=O\left(\|M N\|^{n+\alpha}\right), \text { uniformly } .
$$


This lemma is, after a change of variable, an immediate consequence of Theorem 6I in [C].

\section{Proof of $\mathscr{P}(\mathbf{1})$}

The proof of $\mathscr{P}(1)$ exhibits the main ingredient used to obtain global regularity from partial regularity, namely Taylor's formula at $M, P$, and $Q$ simultaneously, via the projected functions. Since $f$ is $\mathscr{C}^{1, \alpha}$ along the leaves, we obtain

$$
\begin{aligned}
f(R)-f(M) & =\nabla_{s}^{M} f_{M, s}^{M}(M) \cdot \Pi_{s}^{M}(\mathbf{M P})+\nabla_{u}^{M} f_{P, u}^{M}\left(\tilde{\Pi}_{u}^{M}(P)\right) \cdot \Pi_{u}^{M}(\mathbf{P R})+O\left(\|\mathbf{R M}\|^{1+\alpha}\right) \\
& =\nabla_{u}^{M} f_{M, u}^{M}(M) \cdot \Pi_{u}^{M}(\mathbf{M Q})+\nabla_{s}^{M} f_{Q, s}^{M}\left(\tilde{\Pi}_{s}^{M}(Q)\right) \cdot \Pi_{s}^{M}(\mathbf{Q R})+O\left(\|\mathbf{R M}\|^{1+\alpha}\right) .
\end{aligned}
$$

Assume $\alpha \leqq \varepsilon$. Since the partial gradients are bounded, Lemma 1 yields,

$$
\begin{aligned}
& {\left[\nabla_{s}^{M} f_{M, s}^{M}(M)-\nabla_{s}^{M} f_{Q, s}^{M}\left(\tilde{\Pi}_{s}^{M}(Q)\right)\right] \cdot \Pi_{s}^{M}(\mathbf{M P})} \\
& \quad=\left[\nabla_{u}^{M} f_{M, u}^{M}(M)-\nabla_{u}^{M} f_{P, u}^{M}\left(\tilde{\Pi}_{u}^{M}(P)\right)\right] \cdot \Pi_{u}^{M}(\mathbf{P Q})+O\left(\|\mathbf{R M}\|^{1+\alpha}\right) .
\end{aligned}
$$

If $\|\mathbf{M P}\|=\|\mathbf{Q M}\|^{1+\eta}$, this implies

$$
\left[\nabla_{u}^{M} f_{M, u}^{M}(M)-\nabla_{u}^{M} f_{P, u}^{M}\left(\tilde{\Pi}_{u}^{M}(P)\right)\right] \cdot \Pi_{u}^{M}(\mathbf{M Q})=O\left(\|\mathbf{R M}\|^{(\alpha \wedge \eta)+1}\right) .
$$

If $\alpha<\eta$, it follows

$$
\left[\nabla_{u}^{M} f_{M, u}^{M}(M)-\nabla_{u}^{M} f_{P, u}^{M}\left(\tilde{\Pi}_{u}^{M}(P)\right)\right] \cdot \Pi_{u}^{M}(\mathbf{M Q})=O\left(\|\mathbf{Q M}\|\|\mathbf{P M}\|^{\alpha / 1+\eta}\right),
$$

so that

$$
\nabla_{u}^{M} f_{M, u}^{M}(M)-\nabla_{u}^{M} f_{P, u}^{M}\left(\tilde{\Pi}_{u}^{M}(P)\right)=O\left(\|\mathbf{P M}\|^{\alpha / 1+\eta}\right) .
$$

This clearly implies that $\nabla_{u} f$ is Hölder along the leaves of $\mathscr{F}_{s}$. Since it is also Hölder along the leaves of $\mathscr{F}_{u}$ it is globally Hölder. Similarly for $\nabla_{s} f$. It follows easily that $f$ is $\mathscr{C}^{1}$. Moreover there exists a $\mathrm{GL}_{m}(\mathbb{R})$-valued Hölder function $M \rightarrow \Psi_{M}$, such that $\nabla f(M)=\Psi_{M}\left(\nabla_{s} f(M)+\nabla_{u} f(M)\right)$. Therefore $f$ is $\mathscr{C}^{1, \beta}$ for some $\beta>0$ and $\mathscr{P}(1)$ is proved.

\section{Proof of $\mathscr{P}(n) \rightarrow \mathscr{P}(n+1)$}

Suppose $f$ is $\mathscr{C}^{n, \alpha}$, and $\mathscr{C}^{n+1, \alpha}$ along the leaves of $\mathscr{F}_{s}$ and $\mathscr{F}_{u}$, with bounds. Then we want to show that its partial derivatives of order 1 are $\mathscr{C}^{n, \beta}$ along the leaves of $\mathscr{F}_{s}$ and $\mathscr{F}_{u}$. By $\mathscr{P}(n)$ this will imply that they are globally $\mathscr{C}^{n, \gamma}$ for some $\gamma>0$, so that $f$ will be $\mathscr{C}^{n+1, \gamma}$.

Let $M$ be a point, $P_{n, M}$ be the Taylor polynomial of order $n$ at $M$ and $g=f-P_{n, M}$.

1. Since $f$ is $\mathscr{C}^{n+1}$ along the leaves with bounded $n+1-$ st derivatives, Taylor's formula implies

$$
\begin{aligned}
g(R)-g(M) & =\sum_{j=1}^{n}\left[\nabla_{u}^{M \otimes j} g_{P, u}^{M}\left(\widetilde{\Pi}_{u}^{M}(P)\right)\right]\left[\frac{\left(\Pi_{u}^{M}(\mathbf{P R})\right)^{\otimes j}}{j !}\right]+O\left(\|\mathbf{R M}\|^{n+1}\right) \\
& =\sum_{k=1}^{n}\left[\nabla_{s}^{M \otimes k} g_{Q, s}^{M}\left(\tilde{\Pi}_{s}^{M}(Q)\right)\right]\left[\frac{\left(\Pi_{s}^{M}(\mathbf{Q R})\right)^{\otimes k}}{k !}\right]+O\left(\|\mathbf{R M}\|^{n+1}\right)
\end{aligned}
$$


Since $g$ is $\mathscr{C}^{n, \alpha}$ and all its derivatives up to order $n$ vanish at $M$,

$$
\begin{aligned}
& \nabla_{u}^{M \otimes j} g_{P, u}^{M}\left(\tilde{\Pi}_{u}^{M}(P)\right)=O\left(\|\mathbf{M P}\|^{n-j+\alpha}\right), \\
& \nabla_{s}^{M \otimes k} g_{Q, s}^{M}\left(\tilde{\Pi}_{s}^{M}(Q)\right)=O\left(\|\mathbf{M P}\|^{n-k+\alpha}\right) .
\end{aligned}
$$

Also, by Lemma 1 , if $c\|M P\|^{1+n} \leqq\|M Q\| \leqq C\|M P\|^{1 / 1+n}$

$$
\begin{aligned}
& \Pi_{u}^{M}(\mathbf{P R})^{\otimes j}-\Pi_{u}^{M}(\mathbf{M Q})^{\otimes j}=O\left(\|\mathbf{Q M}\|^{j+\varepsilon}\right), \\
& \Pi_{s}^{M}(\mathbf{Q R})^{\otimes k}-\Pi_{s}^{M}(\mathbf{M P})^{\otimes k}=O\left(\|\mathbf{P M}\|^{k+\varepsilon}\right) .
\end{aligned}
$$

From (4.1)-(4.5) we obtain

$$
\begin{aligned}
\sum_{j=1}^{n} & {\left[\nabla_{u}^{M \otimes j} g_{P, u}^{M}\left(\tilde{\Pi}_{u}^{M}(P)\right)\right] \cdot\left[\frac{\left.\Pi_{u}^{M}(\mathbf{M Q})\right)^{\otimes j}}{j !}\right] } \\
= & \sum_{k=1}^{n}\left[\nabla_{s}^{M \otimes k} g_{Q, s}^{M}\left(\tilde{\Pi}_{s}^{M}(Q)\right)\right] \cdot\left[\frac{\Pi_{s}^{M}(\mathbf{M P})^{\otimes k}}{k !}\right]+O\left(\|\mathbf{R} \mathbf{M}\|^{n+\gamma}\right),
\end{aligned}
$$

where $\gamma=1 \wedge \alpha+\varepsilon$

In the following lemma, $S_{j, d_{u}}$ denotes the space of symmetric $j$-linear forms on $\mathbb{R}^{d_{u}}$, and similarly for $S_{k, d_{s}}$.

Lemma 3. Let $g_{j}: \mathbb{R}^{d_{s}} \rightarrow S_{j, d_{u}}, j \in[1, n]$ and $f_{k}: \mathbb{R}^{d_{u}} \rightarrow S_{k, d_{s}}, k \in[1, n]$, be such that $g_{j}(z)=O\left(|z|^{n-j+\alpha}\right)$ and $f_{k}(z)=O\left(|z|^{n-k+\alpha}\right)$ for some $\alpha>0$. If for $y \in \mathbb{R}^{d_{s}}$ and $x \in \mathbb{R}^{d_{u}}$, and when

$$
\begin{gathered}
c|y|^{1+\eta} \leqq|x| \leqq C|y|^{1 / 1+\eta},|x| \leqq 1,|y| \leqq 1 \\
\sum_{1 \leqq j \leqq n} g_{j}(y) x^{\otimes j}+\sum_{1 \leqq k \leqq n} f_{k}(x) y^{\otimes k}=O\left(|x|+|y|^{n+\beta}\right)
\end{gathered}
$$

for some $\beta \in] \alpha, 1]$, then $g_{j}(z)=O\left(|z|^{n-j+w}\right)$ and $f_{k}(z)=O\left(|z|^{n-k+w}\right)$ for all $w<\beta$.

To prove this lemma, we let

$$
\omega_{j}=\sup \left\{\omega, \omega \in \mathbb{R}, \varlimsup_{|z| \rightarrow 0} \frac{\left|f_{j}(z)\right|}{|z|^{n-j+\omega}}<+\infty\right\}
$$

and we define $\omega_{k}^{\prime}$ similarly. We shall show by contradiction that $\inf \left\{\omega_{j}, \omega_{k}^{\prime}\right.$, $j \in[1, n], k \in[1, n]\} \geqq \beta$. Notice that by definition of $\omega_{j}$, or $\omega_{k}^{\prime}$,

$$
\varlimsup_{\substack{|y| \rightarrow 0 \\|x|=|y|^{1+\xi}}} \frac{\left|g_{j}(y) x^{\otimes j}\right|}{|y|^{n+\xi j+v}}
$$

is zero if $v<\omega_{j}$ and infinite if $v>\omega_{j}$, for all $\xi \in\left[\frac{-\eta}{1+\eta}, \eta\right]$, and similarly for

$$
\varlimsup_{\substack{|y| \rightarrow 0 \\|x|=|y|^{1+\xi}}} \frac{\left|f_{k}(x) y^{\otimes k}\right|}{|y|^{(1+\xi)(n-k+v)+k}}
$$


Suppose that $\inf \left\{\omega_{j}, \omega_{k}^{\prime}\right\}<\beta$. Taking $\xi=0$, we see that (4.7) cannot hold if $\inf \left\{\omega_{j}, \omega_{k}^{\prime}\right\}$ is not attained for two different values of $j$ 's or $k$ 's. Similarly for $\inf \left\{n+\xi j+\omega_{j},(1+\xi)\left(n-k+\omega_{k}^{\prime}\right)+k\right\}$, for all $\xi$ close enough to 0 . Then this implies that there exists one $j_{0}$ and one $k_{0}$ so that $n+\xi j_{0}+\omega_{j_{0}}=(1+\xi)\left(n-k_{0}+\omega_{k_{0}}^{\prime}\right)+k_{0}$ for all $\xi$. This implies $\omega_{j_{0}}=\omega_{k_{0}}^{\prime}=k_{0}+j_{0}-n$. Since the $\omega_{j}$ 's are bigger than $\alpha, \omega_{j_{0}}$ and $\omega_{k_{0}}^{\prime}$ are bigger than 1. Moreover $\omega_{j_{0}}=\inf \left\{\omega_{j}, \omega_{k}^{\prime}\right\}$, which contradicts $\inf \left\{\omega_{j}, \omega_{k}^{\prime}\right\}<\beta$. This proves the lemma. Notice that the constants are uniform in the conclusions of the lemma, given the constants of the assumptions.

From this lemma and (4.6) we see that, if (4.2) and (4.3) hold for some $\alpha<1$, then it holds for all $\omega$ 's less than $1 \wedge \alpha+\varepsilon$. Hence (4.2) and (4.3) hold for all $\alpha<1$.

2. The next step is to show that in (4.6) $O\left(\|\mathbf{R} \mathbf{M}\|^{n+\gamma}\right)$ can be replaced by $O\left(\|\mathbf{R M}\|^{n+1+\xi}\right)$ for some $\xi>0$. To this effect we use Taylor's formula of order $(n+1)$. Since $f$ is $\mathscr{C}^{n+1, \alpha}$ along the leaves we obtain, as for (4.1),

$$
\begin{aligned}
\sum_{j=1}^{n+1}[ & \left.\nabla_{u}^{M \otimes j} g_{P, u}^{M}\left(\tilde{\Pi}_{u}^{M}(P)\right)\right]\left[\frac{\left(\Pi_{u}^{M}(\mathbf{P R})\right)^{\otimes j}}{j !}\right] \\
& +\left[\nabla_{s}^{M \otimes n+1} g_{M, s}^{M}(M)\right]\left[\frac{\left(\Pi_{s}^{M}(\mathbf{M P})\right)^{\otimes n+1}}{n+1 !}\right] \\
= & \sum_{k=1}^{n+1}\left[\nabla_{s}^{M \otimes k} g_{Q, s}^{M}\left(\tilde{\Pi}_{s}^{M}(Q)\right)\right]\left[\frac{\left(\Pi_{s}^{M}(\mathbf{Q R})\right)^{\otimes k}}{k !}\right] \\
& +\left[\nabla_{u}^{M \otimes n+1} g_{M, u}^{M}(M)\right]\left[\frac{\left(\Pi_{u}^{M}(\mathbf{M Q})\right)^{\otimes n+1}}{n+1 !}\right]+O\left(\|\mathbf{R M}\|^{n+1+\alpha}\right) .
\end{aligned}
$$

If we set $\|\mathbf{M Q}\|=\|\mathbf{M P}\|^{1+\mu}$ for some $\mu<\eta$, then we obtain, by (4.2) and (4.3) which are valid for all $\alpha<1$,

$$
\begin{aligned}
{\left[\nabla_{s}^{M \otimes n+1} g_{M, s}^{M}(M)\right]\left[\frac{\left(\Pi_{s}^{M}(\mathbf{M P})\right)^{\otimes n+1}}{n+1 !}\right]=} & {\left[\nabla_{s}^{M \otimes n+1} g_{Q, s}^{M}\left(\tilde{\Pi}_{s}^{M}(Q)\right)\right]\left[\frac{\Pi_{s}^{M}(\mathbf{Q R})^{\otimes n+1}}{n+1 !}\right] } \\
& +O\left(\|\mathbf{R M}\|^{n+1+\alpha}+\|\mathbf{R} \mathbf{M}\|^{n+1+v}\right)
\end{aligned}
$$

for all $v<\mu$. Using (4.4) with $k=n+1$, we see that

$$
\begin{aligned}
& {\left[\nabla_{s}^{M \otimes n+1} g_{M, s}^{M}(M)-\nabla_{s}^{M \otimes n+1} g_{Q, s}^{M}\left(\tilde{\Pi}_{s}^{M}(Q)\right)\right]\left[\frac{\Pi_{s}^{M}(\mathbf{Q R})^{\otimes n+1}}{n+1 !}\right]} \\
& \quad=O\left(\|\mathbf{R M}\|^{n+1+(\alpha \wedge v \wedge \varepsilon)}\right) .
\end{aligned}
$$

This implies, for some $\xi>0$,

$$
\left[\nabla_{s}^{M \otimes n+1} g_{M, s}^{M}(M)-\nabla_{s}^{M \otimes n+1} g_{Q, s}^{M}\left(\tilde{\Pi}_{s}^{M}(Q)\right)\right]=O\left(\|\mathbf{Q M}\|^{\xi}\right) .
$$

A similar estimate holds in the $u$-direction. Together with (4.8) and (4.2)-(4.5) these estimates imply that, in (4.6), $O\left(\|\mathbf{R} \mathbf{M}\|^{n+\gamma}\right)$ can be replaced by $O\left(\|\mathbf{R M}\|^{n+1+\xi}\right)$ for some $\xi>0$.

3. Now we set $\|\mathbf{Q M}\|=\|\mathbf{P M}\|^{1+v}$ for some $v$ small. Then for $j \in[2, n]$, by (4.2),

$$
\left[\nabla_{u}^{M \otimes j} g_{P, u}^{M}\left(\tilde{\Pi}_{u}^{M}(P)\right)\right] \cdot\left[\frac{\left(\Pi_{u}^{M}(\mathbf{M Q})\right)^{\otimes j}}{j !}\right]=O\left(\|\mathbf{R M}\|^{n+1+\mu}\right)
$$


for all $\mu<2 v$, and also if $k \in[1, n-1]$, by (4.3),

$$
\left[\nabla_{s}^{\mathbf{M}}{ }^{\otimes} g_{Q, s}^{M}\left(\tilde{\Pi}_{s}^{M}(Q)\right)\right] \cdot\left[\frac{\left(\Pi_{s}^{M}(\mathbf{M P})\right)^{\otimes k}}{k !}\right]=O\left(\|\mathbf{R M}\|^{n+1+\mu}\right) .
$$

Then (4.6) with $O\left(\|\mathbf{R M}\|^{n+1+\xi}\right)$ instead of $O\left(\|\mathbf{R M}\|^{n+\gamma}\right)$ becomes, if $v \leqq \frac{\xi}{2}$,

$$
\begin{aligned}
{\left[\nabla_{u}^{M} g_{P, u}^{M}\left(\tilde{\Pi}_{u}^{M}(P)\right)\right] \cdot\left[\Pi_{u}^{M}(\mathbf{M Q})\right]=} & {\left[\nabla_{s}^{M \otimes n} g_{Q, s}^{M}\left(\tilde{\Pi}_{s}^{M}(Q)\right)\right] \cdot\left[\frac{\left.\Pi_{s}^{M}(\mathbf{M P})\right)^{\otimes n}}{n !}\right] } \\
& +O\left(\|\mathbf{R} \mathbf{M}\|^{n+1+\mu}\right)
\end{aligned}
$$

for all $\mu<2 v$. We now suppose $\mu$ fixed bigger than $v$.

Observe that (4.9) remains true if $\|\mathbf{Q M}\| \simeq\|\mathbf{P M}\|^{1+v}$.

We fix a unit vector e in $T_{u}^{M}$ and we denote for all $\lambda>0$ small enough by $Q_{\lambda}$ the point of $\mathscr{L}_{u}^{M}$ such that $\Pi_{u}^{M}\left(\mathbf{M} \mathbf{Q}_{\lambda}\right)=\lambda$ e. We let $G: \mathbb{R}_{+} \rightarrow S_{n, d_{s}}$ be defined by $G(\lambda)=\left[\nabla_{s}^{M \otimes n} g_{Q_{\lambda}, s}^{M}\left(\tilde{\Pi}_{s}^{M}\left(Q_{\lambda}\right)\right)\right]$. From (4.9) we obtain, for all $z \in[1,2]$

$$
G(\lambda)-z G\left(\frac{\lambda}{z}\right)=O\left(\|\mathbf{R M}\|^{1+\mu}\right)=\mathrm{O}\left(\lambda^{\frac{1+\mu}{1+v}}\right) .
$$

Therefore $\frac{G(\lambda)}{\lambda}$ has a limit $L$ when $\lambda \rightarrow 0$ and $\frac{G(\lambda)}{\lambda}-L=O\left(\lambda^{\frac{\mu-v}{1+v}}\right)$. We then can rewrite (4.9) as

$$
\left[\nabla_{u}^{M} g_{P, u}^{M} \tilde{\Pi}_{u}^{M}(P)\right] \cdot \mathbf{e}=L \cdot \frac{\left(\Pi_{s}^{M}(\mathbf{M P})\right)^{\otimes n}}{n !}+O\|\mathbf{M P}\|^{n+\mu-v} .
$$

Since $g$ is $\mathscr{C}^{n+1, \alpha}$ on $\mathscr{L}_{s}^{M}$ and all its derivatives up to order $n$ vanish at $M$, for all unit vector $\mathbf{f}$ of $T_{s}^{M}$,

$$
\left[\nabla_{s}^{M} g_{P, s}^{M} \tilde{\Pi}_{s}^{M}(P)\right] \cdot \mathbf{f}=L^{\prime} \cdot \frac{\left(\Pi_{s}^{M}(\mathbf{M P})\right)^{\otimes n}}{n !}+O\|\mathbf{M P}\|^{n+\alpha}
$$

Notice that $\nabla g(P)$ can be reconstructed from $\nabla_{u}^{M} g_{P, u}^{M}\left(\tilde{\Pi}_{u}^{M}(P)\right)+\nabla_{u}^{M} g_{P, s}^{M}\left(\tilde{\Pi}_{s}^{M}(P)\right)$ $=X(M, P)$. More precisely if $\Psi_{M, P}$ is the isomorphism of $\mathbb{R}^{d_{u}+d_{s}}$ such that $\Psi_{M, P}(X(M, P))=\nabla_{g}(P)$, then

$$
\Psi_{M, M}=I, \quad \text { and } \quad\left\|\Psi_{M, P}-I\right\|=O\left(\|\mathbf{M P}\|^{\delta}\right) \text { for some } \delta>0 .
$$

From (4.10) and (4.11) we obtain

$$
\nabla g(p)=\tilde{L} \cdot \frac{\Pi_{s}^{M}(\mathbf{M P})^{\otimes n}}{n !}+O\|\mathbf{M P}\|^{n+\alpha \wedge \delta}
$$

where $\tilde{L}$ is a symmetric $n$-linear application valued in $\mathbb{R}^{d_{s}+d_{u}}$. From (4.12) it follows that $\nabla f=\nabla g+\nabla P_{n, M}$ satisfies the assumptions of Lemma 2. By Lemma $2 \nabla f$ is $\mathscr{C}^{n, \alpha}$ along the leaves of $\mathscr{F}_{s}$, and also of $\mathscr{F}_{u}$, if $\alpha \leqq \delta$. By $\mathscr{P}(n), \nabla f$ is $\mathscr{C}^{n, \beta}$ for some $\beta>0$, so that $f$ is $\mathscr{C}^{n+1, \beta}$. This concludes the proof of the theorem. 


\section{References}

[C] Campanato, S.: Proprietá dí una famiglia dí spazi funzionali. Ann. Sc. Norm. Super. Pisa 18, 137-160 (1964)

[L] de la Llave, R.: Invariants for smooth conjugacy of hyperbolic systems. II (submitted to Commun. Math. Phys.)

[LMM] de la Llave, R., Marco, J.M., Moriyon, R.: Canonical perturbation theories for Anosov's systems and regularity results for the Livsic's Cohomology Equation. Ann. Math. to appear. See also Bull. Am. Math. Soc. 12, 1, 91-94 (1985)

[MM] Marco, J.M., Moriyon, R.: Invariants for smooth conjugacy of hyperbolic systems. I (submitted to Commun. Math. Phys.)

Communicated by J. Mather

Received March 20, 1986 
